Canadian Oncology

Nursing Journal

Revue canadienne

de soins infirmiers

en oncologie

Volume 30, Issue 2 • Spring 2020

elSSN: 2368-8076 


\title{
Élaboration d'un énoncé de position national sur la navigation des patients atteints de cancer au Canada
}

\author{
par Kristen R. Haase, Jennifer Anderson, Andrea Knox, Myriam Skrutkowski, Beth Snow, Lesley Moody, Zenith Pool, Kristina \\ Vimy et Linda Watson
}

\section{RÉSUMÉ}

Au Canada, le paysage des soins du cancer évolue et les infirmières en oncologie sont appelées à jouer de nouveaux rôles afin d'améliorer l'expérience des patients et des familles et de répondre à leurs besoins toujours changeants. L'un de ces rôles, la navigation des patients atteints de cancer (NPC), vise principalement à coordonner les soins centrés sur la personne et à guider les patients dans le système de santé. Dans plusieurs provinces et territoires du Canada, la navigation est devenue essentielle pour offrir des soins de grande qualité aux patients cancéreux. Depuis 2016, des chefs de file des soins infirmiers oncologiques se réunissent au sein d'un groupe national pour synthétiser leur compréhension de la navigation des patients en contexte canadien afin d'élaborer un énoncé de position national sur la NPC. Dans le présent article, nous analyserons l'historique de l'élaboration de lénoncé de position sur la NPC que publiera prochainement l'Association canadienne des infirmières en oncologie (ACIO/CANO). Nous analyserons également les commentaires des participants aux ateliers tenus dans le cadre des congrès de 2016, 2017 et 2018, de même que les comptes rendus des réunions du groupe de travail national qui ont eu lieu pendant cette même période. Le présent article se veut un sommaire historique concis de l'évolution de la NPC au Canada, ainsi qu'un modèle pour les autres groupes aspirant à rédiger un énoncé de position consensuel.

\author{
AUTEURES \\ Kristen R. Haase, inf. aut., Ph.D. \\ Jennifer Anderson, inf. aut. M.Sc.inf., CSIO(C) \\ Andrea Knox, inf. aut., B.Sc.inf, CSIO(C) \\ Myriam Skrutkowski, inf. aut., M.Sc(C), CSIO(C) \\ Beth Snow, inf. aut., B.Sc.inf. \\ Lesley Moody, M.B.A., Ph.D. \\ Zenith Pool, inf. aut., ICSP \\ Kristina Vimy, inf. aut., M.Sc.inf., CSIO(C) \\ Linda Watson, inf. aut., Ph.D., CSIO(C)
}

DOI:10.5737/236880763028392
L 'arrivée de la navigation des patients atteints de canLcer (NPC) dans le paysage des soins infirmiers oncologiques est venue modifier, au cours des vingt dernières années, la gestion et la coordination des soins (Pedersen et Hack, 2010). Bien que la NPC soit organisée différemment d'un bout à l'autre du pays, l'objectif commun est de guider les patients dans le système de santé. La navigation des patients atteints de cancer se définit comme « un processus proactif intentionnel de collaboration avec une personne et sa famille pour les guider dans le labyrinthe des traitements, des services et des obstacles potentiels tout au long de l'expérience globale du cancer » (Partenariat canadien contre le cancer, 2010, p. 5). Les recherches antérieures ont montré que la navigation prodiguée par une infirmière spécialisée en oncologie produit des résultats positifs pour les patients, les familles, les proches aidants, l'équipe de soins interdisciplinaire, les organisations de soins et le système de santé (Campbell, Craig, Eggert et Bailey-Dorton, 2010; Cantril et Haylock, 2013; Case, 2011; McMullan, 2006; Pedersen, Hack, McClement et Taylor-Brown, 2014; Seek et Hogle, 2007). Par exemple, dans un milieu communautaire de soins oncologiques aux ÉtatsUnis, Campbell et collaborateurs (2010) ont constaté une plus grande satisfaction par rapport aux soins et moins de barrières d'accès; en Alberta, une évaluation provinciale du recours aux infirmières pivots en oncologie a conduit à des résultats analogues (Watson, Vimy, Anderson, Champ et DeIure, 2016).

En 2016, le travail d'élaboration d'un énoncé de position national sur la NPC a pris son envol. Les efforts visaient alors à mieux comprendre les multiples définitions du rôle de NPC au Canada, cette compréhension commune pouvant servir à orienter les pratiques de l'Association canadienne des infirmières en oncologie (ACIO/CANO). Nous voulions nous réunir et synthétiser les avis de toutes les provinces et territoires du Canada sur la NPC afin d'élaborer un énoncé de position permettant de situer la navigation dans la pratique des soins infirmiers oncologiques. Le présent article décrit le processus pluriannuel de consultation nationale mené dans le but de créer l'énoncé de position national qui orientera la NPC au Canada.

\section{CONTEXTE - ÉVOLUTION DE LA NAVIGATION AU CANADA}

Dans les soins du cancer, la navigation des patients s'est imposée comme un rôle important pour mettre les patients en 
lien avec les services de santé appropriés le plus rapidement et le plus simplement possible (Freund, 2011; Pratt-Chapman, Simon, Patterson, Risendal et Patierno, 2011). Le rapport sur la navigation du Partenariat canadien contre le cancer (PCCC) expose trois types d'approches qui peuvent être utilisées seules ou en combinaison, selon les besoins des patients (Partenariat canadien contre le cancer, 2010). En premier lieu, le rapport mentionne la navigation professionnelle, confiée à une infirmière ou à un travailleur social; enracinée dans la hiérarchie institutionnelle, elle est souvent rattachée à un hôpital en particulier, ce qui permet aux intervenants pivots de travailler avec les médecins et les autres professionnels de la santé pour coordonner et gérer les soins. Vient ensuite la navigation par les pairs, prodiguée par des bénévoles qui peuvent offrir des conseils aux patients atteints de cancer en se fondant sur leur expérience de la maladie, ou celle de leur famille. Enfin, la navigation virtuelle permet de combiner les principes de la navigation professionnelle et de la navigation par les pairs grâce à la technologie.

Au Canada, les programmes de NPC ont fait leur apparition il y a près de vingt ans. Le premier du genre a été lancé en 2001 par Action cancer Nouvelle-Écosse. En 2005, le Québec a mis en place son programme d'infirmière pivot en oncologie (IPO) (ministère de la Santé et des Services sociaux (MSSS), 2005). Par la suite, de nombreuses provinces ont implanté différents modèles de programmes de NPC. Ces premiers programmes n'ont cessé d'évoluer depuis pour suivre les progrès de la navigation en général et s'adapter aux changements apportés aux soins des patients partout au pays.

En 2010, le PCCC a publié un rapport intitulé « Guide de mise en œuvre de la navigation dans les soins contre le cancer », un document important pour la navigation des patients atteints de cancer au Canada. Tout en décrivant plusieurs types de navigation, ce rapport du PCCC présentait également des stratégies de mise en œuvre et illustrait les retombées potentielles pour le système de santé. Dès lors, les programmes d'infirmières pivots en oncologie déjà en place ont été perfectionnés, et de nouveaux programmes ont vu le jour dans plusieurs provinces et territoires du pays. Les infirmières en oncologie étaient particulièrement bien placées pour jouer ce rôle, puisqu'elles s'occupaient déjà de la majorité des responsabilités de navigation du système de santé inscrites dans la définition du PCCC. Les infirmières en oncologie se doivent d'avoir une connaissance approfondie des effets physiologiques du traitement et du diagnostic, des conséquences psychologiques de la maladie, et des services de soins du cancer sur toute la trajectoire de la maladie (Association canadienne des infirmières en oncologie, 2001; Ferrell, McCabe et Levit, 2013; ministère de la Santé et des Services sociaux, 2008). Si l'on tient compte de la définition de la navigation et de l'objectif commun que constituent les soins centrés sur le patient, ces aptitudes, adjointes à leurs compétences générales, placent les infirmières en oncologie dans une position privilégiée pour jouer le rôle d'intervenant pivot.

Depuis le début des années 2000, Fillion et collaborateurs ont mené des recherches majeures sur les infirmières pivots en oncologie. Dans leurs premiers travaux, ils décrivaient l'infirmière pivot comme une personne dont le rôle consistait à coordonner la complexité des soins et des traitements médicaux, à déterminer les besoins des patients et à faciliter l'accès aux ressources communautaires en employant une approche holistique et centrée sur le patient (Doll et al., 2007; Fillion et al., 2009; Fillion et al., 2006). Les recherches qualitatives subséquentes, réalisées auprès des infirmières pivots du Québec, décrivaient quant à elles des interventions englobant les besoins pratiques, informationnels, émotionnels, psychosociaux, physiques et spirituels (Hébert et Fillion, 2011). Les autres travaux de Fillion et collaborateurs ont mené à la création d'un cadre de navigation professionnelle pour les infirmières en oncologie du Canada (Fillion et al., 2012). Ce cadre comprend deux volets principaux et de multiples sous-domaines : 1) faciliter la continuité des soins, notamment la continuité de l'information, la continuité de la gestion et la continuité de la relation; 2) encourager la responsabilisation du patient et de sa famille, notamment par l'adaptation active, l'autogestion du cancer, et les soins de soutien. Dans les recherches qui ont suivi, les principales compétences de la navigation professionnelle ont été mises en lien avec certains des domaines clés de la pratique infirmière en oncologie : 1) fournir de l'information, 2) dispenser des soins de soutien et un appui émotionnel, et 3) faciliter la coordination et la continuité des soins (Cook et al., 2013). Jusqu'en 2016, ce sont ces travaux qui ont servi de pierre angulaire pour encadrer la NPC en contexte canadien.

\section{Place de la navigation dans l'ACIO/CANO}

Avec l'évolution et la diffusion des programmes de NPC au pays, de plus en plus de membres de l'ACIO/CANO veulent comprendre le portrait de la NPC, ainsi que la place qu'elle occupe dans le vaste ensemble des tâches accomplies par les infirmières en oncologie. En outre, ce sont déjà les infirmières spécialisées en oncologie qui prennent en charge la majorité de ces rôles et la mission de l'ACIO/CANO est justement de promouvoir l'excellence des soins infirmiers en oncologie par la pratique, l'éducation, la recherche et le leadership, au bénéfice de tous les Canadiens. La direction de l'ACIO/CANO a donc jugé qu'il était important de comprendre l'expérience des infirmières spécialisées en oncologie responsable de la NPC et de déterminer si l'association devait mettre en place d'autres structures ou mesures de soutien pour appuyer les infirmières dans ce rôle.

Les discussions officielles se sont ouvertes au congrès 2016 de l'ACIO/CANO à Calgary, où des infirmières de partout au pays ont pris part à un atelier pour discuter de la situation de la NPC au Canada. Les participants à l'atelier ont clairement exprimé l'importance d'élaborer un énoncé de position pour guider l'évolution des rôles de NPC au Canada. À la suite des discussions en atelier, un groupe de travail national a été mis sur pied pour rédiger la version préliminaire de l'énoncé de position sur la NPC de l'ACIO/CANO. D'autres ateliers ont eu lieu aux congrès 2017 et 2018, ce qui a permis de retravailler, peaufiner et finaliser l'énoncé. 
Nous présentons ci-après la genèse de cette initiative pancanadienne et sa transformation au fil du temps. Suivra une analyse approfondie de notre travail, qui rend compte du cheminement de l'énoncé de position sur la navigation (figure 1).

\section{6 : Première réunion au congrès de l'ACIO/CANO}

En octobre 2016, les membres de l'ACIO/CANO et les représentants des soins infirmiers en oncologie du pays ont été invités à participer à une conversation nationale sur la NPC dans le cadre du congrès annuel de l'ACIO/CANO. Le but de cette conversation était de comprendre le rôle de la NPC au Canada et le rôle qu'y jouent les infirmières. Le premier courriel d'invitation a été envoyé à 39 leaders des soins infirmiers oncologiques représentant chaque province et territoire. On les encourageait à assister à l'événement et, s'ils ne pouvaient être présents, à y déléguer une personne en leur nom. Le résumé de l'atelier était disponible dans le programme du congrès de l'ACIO/CANO; l'atelier était ouvert à tous les membres de l'ACIO/CANO et à toutes les personnes présentes au congrès. Trente-neuf personnes ont assisté à la première table ronde.

Les discussions portaient principalement sur l'état actuel de la contribution des infirmières en oncologie à la NPC et sur l'amélioration de l'accès aux services de navigation partout au pays (voir au tableau 1 le résumé de l'état de la navigation au
Canada en 2016 rapporté par les participants à l'atelier). De riches discussions ont eu lieu lorsque les infirmières présentes à la rencontre ont abordé la pression que subit actuellement le système de santé à cause du nombre croissant de patients et des contraintes budgétaires, ce qui laisse fréquemment les patients dans le besoin. Souvent, les infirmières de chevet n'ont pas les connaissances qu'il faut, le temps nécessaire ou la latitude suffisante dans leur définition de tâche pour répondre aux besoins complexes des patients. Les participants ont souligné le fait que, dans de nombreuses provinces canadiennes, la navigation est vue comme un élément clé d'un système intégré de soins du cancer et que l'amélioration des services de navigation optimise la prestation des soins centrés sur la personne. Toutefois, les participants ont aussi fait remarquer que l'organisation et le développement des rôles et des programmes de navigation, tant provinciaux que pancanadiens, sont quelque peu informels, c'est-à-dire qu'ils semblent avoir vu le jour en réponse à des problèmes régionaux bien particuliers plutôt que de façon coordonnée et organisée. Par exemple, certaines provinces offrent des programmes de NPC pour certains types de cancer particuliers (ex. cancer du sein ou cancer gastro-intestinal), tandis que d'autres ont des programmes axés sur la prestation de soins à des moments précis de la trajectoire du

\section{Évolution de l'énoncé de position sur la navigation}

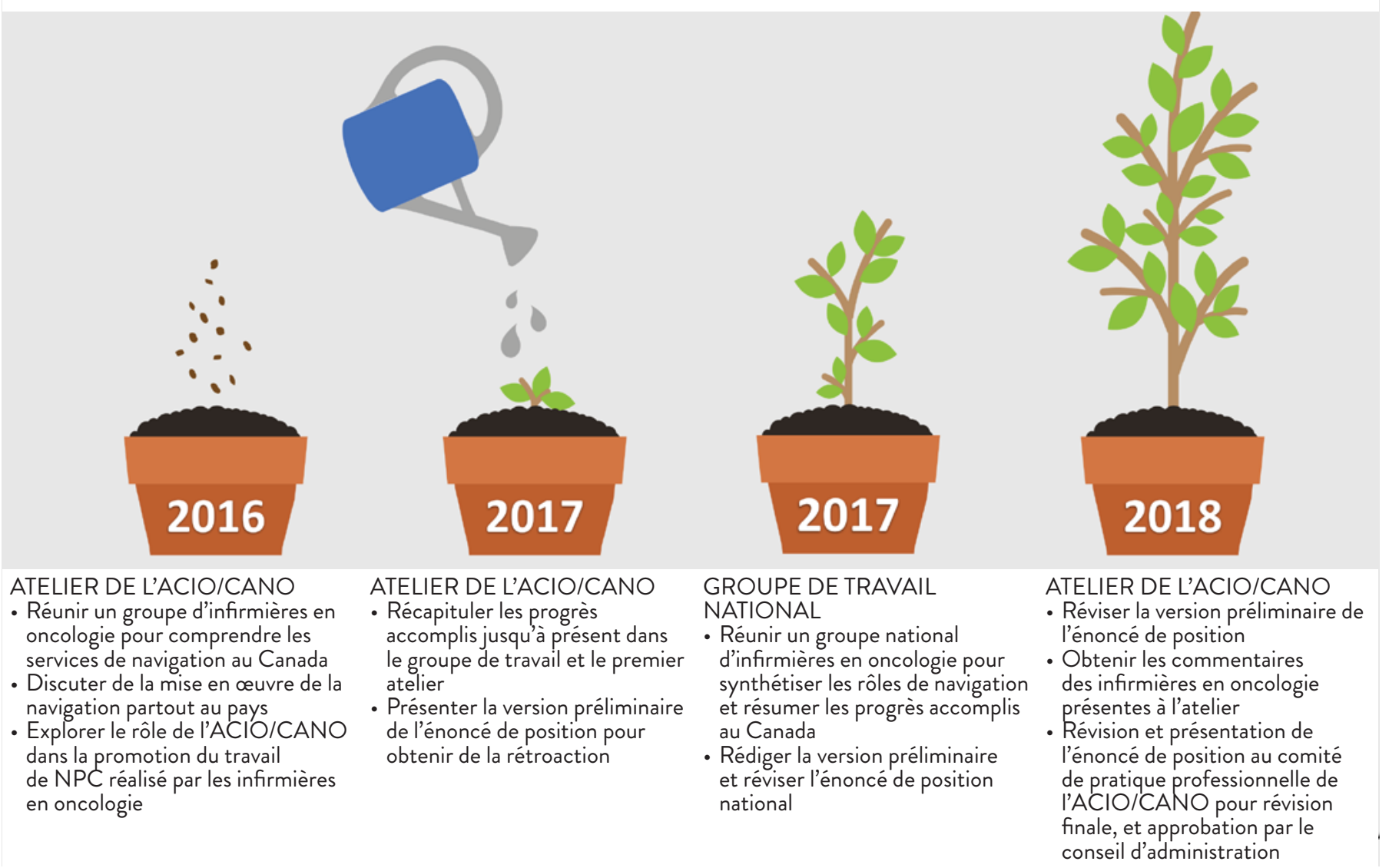

Figure 1 : Évolution de l'énoncé de position sur la navigation, de 2016 à aujourd'hui (créé avec presentationgo.com) 


\begin{tabular}{|c|c|}
\hline $\begin{array}{l}\text { Colombie- } \\
\text { Britannique }\end{array}$ & $\begin{array}{l}\text { II n'existe aucun système pour coordonner les rôles de navigation confiés aux infirmières; on veut que toutes les infirmières en oncologie soient } \\
\text { capables de s'occuper de la navigation, quel que soit leur rôle. } \\
\text { Nous devons examiner les modèles de soins afin de nous assurer que les infirmières interviennent au bon endroit dans le système de soins du } \\
\text { cancer pour répondre aux besoins des patients, et nous devons soutenir toute la portée de leur pratique, et non se contenter d'ajouter une autre } \\
\text { couche avec ce rôle de " pivot ". } \\
\text { Les IP rattachées à des groupes de cancer et certaines infirmières en soins primaires pourraient combler les lacunes dans les soins; des infirmières } \\
\text { en cancer du sein l'ont fait par le passé. }\end{array}$ \\
\hline Alberta & $\begin{array}{l}3 \text { modèles : 1) généraliste - du diagnostic aux soins de fin de vie; tous les sites - dans les collectivités rurales ou isolées qui ont un centre de } \\
\text { traitement du cancer. Gestion et organisation provinciale; } 2 \text { ) intervenant pivot autochtone en poste au } \mathrm{CCl} \text {, mais le financement provient } \\
\text { d'organismes de santé autochtones, qui travaillent en étroite collaboration avec le programme communautaire de navigation des patients en } \\
\text { oncologie mentionné précédemment; 3) cancer du sein - des présomptions de cancer à la première consultation en chirurgie - trois programmes } \\
\text { de ce type pour le cancer du sein dans les centres urbains. } \\
\text { Certains programmes ponctuels dans d'autres centres, notamment des intervenants pivots pour le cancer du sein à Misericordia, pour le cancer } \\
\text { des ovaires au Royal Alex d'Edmonton et intervenants pivots pour les patients hospitalisés en hématologie. }\end{array}$ \\
\hline Manitoba & $\begin{array}{l}\text { Les intervenants pivots voient le patient avant le début du traitement et lui offrent du soutien au besoin. Le système provincial doit se transformer } \\
\text { pour faire de la place aux équipes de navigation, à la création de " points de service » et à la collaboration avec les soins primaires. Au départ, les } \\
\text { rôles ont été mis en place en milieu rural, mais sont maintenant présents en milieu urbain. } \\
\text { Dans les centres urbains, l'accent est mis sur la réduction du nombre de visites aux urgences et également sur les patients dont le cancer est } \\
\text { avancé et qui attendent un rendez-vous en clinique; les intervenants pivots peuvent les visiter en personne ou les contacter par téléphone. Ils } \\
\text { travaillaient en étroite collaboration avec les soins palliatifs, les cliniques de diagnostic rapide, les médecins de famille (bon réseau dans la province). }\end{array}$ \\
\hline Ontario & $\begin{array}{l}\text { Il existe de nombreux types d'intervenants pivots travaillant dans toute une variété de milieux, de groupes, de points de la trajectoire des soins, etc. } \\
\text { partout dans la province (aucune norme ou structure d'encadrement provinciale). } \\
\text { Programme d'évaluation diagnostique - les rôles varient au sein du programme; certains sont des coordonnateurs administratifs du cheminement des } \\
\text { soins. Des modèles de coordination administrative sont aussi mis à l'essai. ll y a aussi de la navigation par les pairs. } \\
\text { Le rôle dépend principalement de l'organisation; par conséquent, les rôles et responsabilités varient; chaque région et programme d'évaluation } \\
\text { diagnostique est différent; il n'existe pas de modèle directeur provincial. }\end{array}$ \\
\hline $\begin{array}{l}\text { Nouveau- } \\
\text { Brunswick }\end{array}$ & Il existe des services de navigation pédiatrique, mais aucun autre programme de navigation coordonnée. \\
\hline $\begin{array}{l}\text { Île-du-Prince- } \\
\text { Édouard }\end{array}$ & $\begin{array}{l}\text { Toutes les infirmières en milieu oncologique font de la navigation. Une infirmière est spécialement désignée pour jouer le rôle d'intervenant pivot; } \\
\text { elle possède de l'expérience en santé mentale; elle travaille au principal centre de Charlottetown, mais se rend à Summerside un jour par semaine. } \\
\text { La principale lacune dans les soins aux patients est la période précédant la mise en lien avec le centre de cancérologie. Le rôle de navigation, pour l'instant, } \\
\text { n'est pas encore bien connu et il continue d'évoluer. Les soins de suivi (aux survivants) sont aussi en évolution. }\end{array}$ \\
\hline $\begin{array}{l}\text { Nouvelle- } \\
\text { Écosse }\end{array}$ & $\begin{array}{l}\text { Les intervenants pivots sont des infirmières autorisées. Neuf en oncologie adulte et } 8 \text { en oncologie pédiatrique; « généraliste "; les infirmières en } \\
\text { milieu communautaire tendent à suivre les patients pendant toute la durée des soins, et pendant toute leur expérience du cancer si l'intervenant } \\
\text { pivot responsable du patient en traitement actif se retire. } \\
\text { Les infirmières pivots du centre de cancérologie d'Halifax s'occupent surtout des cancers de la tête et du cou, et deux se consacrent au cancer du } \\
\text { sein. Les infirmières cliniciennes spécialisées prennent le relais une fois que le patient arrive au centre. }\end{array}$ \\
\hline Terre-Neuve & $\begin{array}{l}\text { Les membres de l'équipe de navigation des patients atteints de cancer sont présents partout dans la province. Il s'agit d'infirmières autorisées } \\
\text { en oncologie qui sont sensibles aux différences culturelles et qui sont hautement qualifiées; elles sont disponibles dès la présomption de cancer } \\
\text { pour aider les patients et les familles, les professionnels de la santé et les partenaires communautaires à s'assurer qu'ils ont l'information et les } \\
\text { connaissances nécessaires pour prendre les meilleures décisions possible au sujet des soins. }\end{array}$ \\
\hline
\end{tabular}


cancer (ex. avant le diagnostic ou la chirurgie); d'autres encore offrent un libre accès aux services de navigation sur toute la trajectoire des soins. De l'avis des participants, ces différences structurelles entre les programmes ont des répercussions sur la mise en pratique du rôle de navigation et sur l'atteinte des résultats au niveau du système.

Nous savons que la portée, l'objectif et la conception des programmes de navigation diffèrent d'une région à l'autre et influencent les résultats auxquels on pourrait s'attendre, mais les participants ont insisté sur le fait que les rôles de NPC se ressemblaient énormément malgré tout. Cependant, en dépit des similitudes, il demeure nécessaire de clarifier le tout. En général, les participants s'entendaient pour dire que les infirmières pivots amélioraient les soins centrés sur la personne en adaptant leurs interventions au contexte et aux besoins uniques du patient.

De ces discussions de groupe ont émergé huit recommandations clés, résumées au tableau 2. La principale rappelle l'importance de considérer la navigation comme l'une des dimensions du rôle de l'infirmière spécialisée en oncologie au même titre que l'évaluation complète de l'état de santé, la relation thérapeutique, la prise en charge des symptômes, l'enseignement et l'encadrement, la prise de décision et la défense des droits des patients, la pratique professionnelle et le leadership (Association canadienne des infirmières en oncologie, 2001). Selon les besoins des patients, leur répartition et le niveau d'interaction, l'infirmière spécialisée en oncologie peut jouer un rôle à la fois d'avant et d'arrière-plan dans la NPC. Parmi les autres suggestions des participants, mentionnons entre autres la nécessité de faire davantage de recherche sur les expériences des patients et les effets de la navigation au Canada, l'importance pour l'ACIO/CANO d'élaborer un énoncé de position sur la navigation, et un intérêt marqué envers la création d'un groupe d'intérêt spécial sur la NPC.

\section{7 : Atelier du congrès de l'ACIO/CANO}

À la suite des recommandations formulées à l'atelier de 2016, un autre atelier a été organisé au congrès de l'ACIO/ CANO en 2017. À cette occasion, nous avons revisité les enjeux de la navigation qui avaient dominé la discussion en 2016. Nous avons également exploré la compréhension commune de la NPC partout au pays, ses bénéfices ajoutés et les compétences dont les infirmières pivots ont besoin pour jouer leur rôle (la discussion est résumée au tableau 3). En somme, le consensus était que les infirmières en oncologie se trouvaient dans une position idéale pour se charger de la navigation et guider les patients tout au long de la trajectoire du cancer. Les participants ont expliqué que la NPC n'est pas uniquement le fait des infirmières : au Canada, les programmes de navigation sont aussi menés par des travailleurs sociaux et des bénévoles. Les participants ont néanmoins fait valoir la valeur ajoutée qu'apportent les infirmières au rôle de navigation, étant donné leur connaissance approfondie des défis physiques, psychosociaux et systémiques que vivent les patients atteints de cancer. Selon les participants, les compétences requises pour la NPC comprennent la capacité de gérer des états de santé complexes et changeants, de même que l'habileté à comprendre les besoins particuliers de la population cible et à y répondre aux bons moments. De l'avis de la plupart des participants, il n'est pas simple de définir concrètement la navigation; de plus, la définition et la mise en pratique de la NPC adoptées au sein d'une organisation influencent les compétences requises pour chaque intervenant pivot.

\section{7 : Création d'un groupe de travail national}

En 2017, un groupe de travail national a été formé à la suite des discussions de l'atelier et de la recommandation de préparer un énoncé de position. Un courriel d'invitation à se joindre à un groupe de travail national sur les infirmières pivots en oncologie a été envoyé à 85 personnes ayant participé aux ateliers du congrès, manifesté leur intérêt ou dont le nom figurait sur la liste de sollicitation originale du projet. Douze personnes ont répondu à cette invitation; elles provenaient de : Terre-Neuve, Ontario, Manitoba, Nouvelle-Écosse, Saskatchewan, Alberta, Colombie-Britannique et Québec. En 2018, le groupe de travail s'est réuni quatre fois par téléconférence et a échangé beaucoup de courriels. Les communications et téléconférences du groupe étaient menées et coordonnées par l'auteure principale (Linda Watson), avec l'aide du personnel de l'ACIO/CANO.

À la première téléconférence, en avril 2018, les participants d'un océan à l'autre étaient invités à discuter des aspects fondamentaux de la NPC, à exposer leurs définitions de la navigation, et à présenter les défis liés à l'actualisation et à la mise en œuvre de la navigation dans leur région. Les notes de cette réunion, qui résumaient toute la richesse de la discussion, ont par la suite fait l'objet d'une analyse thématique. Quatre grands thèmes en sont ressortis (Braun et Clarke, 2008) : 1) répondre aux demandes complexes et diverses des patients ayant des besoins variables; 2) assurer la continuité sur toute la trajectoire des soins, de la période précédant le traitement jusqu'à la survivance; 3) répondre aux demandes qu'on ne sait où adresser à cause du flou entre les limites propres à chaque rôle dans l'équipe soignante et régler les tensions causées par les recoupements entre les diverses disciplines; et 4) définir clairement les limites de la NPC. Le tableau 4 présente en détail les thèmes et les définitions.

Cette première rencontre $\mathrm{du}$ groupe de travail a permis aux membres de mettre en commun leurs points de vue sur la navigation et de brosser le portrait pancanadien de la navigation. Avant la réunion suivante, les membres ont reçu les comptes rendus détaillant les thèmes. À l'approche de la remise de la version préliminaire de l'énoncé de position, ces thèmes ont servi de balises pour structurer la rédaction.

Avant la rencontre suivante, les notes ont été distribuées aux membres du groupe, de même que la version préliminaire de l'énoncé de position rédigée par l'auteure principale (Linda Watson). Une liste de questions ayant pour but de clarifier le contenu et l'orientation de l'énoncé de position a également été remise aux membres du groupe de travail, qui devaient commenter l'énoncé avant la rencontre suivante. Voici les questions posées au groupe de travail : 


\section{Tableau 2 : Recommandations de la table ronde de 2016}

1. Axer la conversation sur la navigation intégrée au rôle de l'infirmière spécialisée en oncologie, c'est-à-dire envisager la navigation et la coordination des soins comme une dimension à part entière au même titre que l'évaluation complète de l'état, la relation thérapeutique, la prise en charge des symptômes, à l'enseignement et l'encadrement, la prise de décision et la défense des droits des patients, la pratique professionnelle et le leadership.

2. Examiner la navigation du point de vue du public.

a. Les gens s'inquiètent de l'écart entre les attentes des patients et l'expérience des soins; la navigation peut-elle réduire cet écart?

b. Comment le public et les patients perçoivent-ils la navigation?

3. Mesurer l'influence de la navigation sur les résultats des patients et du système.

4. Reconnaître que le rôle d'intervenant pivot n'enlève rien à celui de l'infirmière spécialisée en oncologie; il est plutôt axé principalement sur la navigation et la coordination des soins, tandis que les autres rôles infirmiers couvrent plusieurs dimensions.

5. Réduire les obstacles au positionnement optimal des infirmières spécialisées en oncologie dans le système de soins du cancer pour répondre aux besoins des patients. Prendre en compte les facteurs structurels, contextuels et autres qui à la base de notre système fragmenté de soins du cancer, ainsi que les rôles de navigation (sinon, la navigation ne sert que de " pansement " pour un système malade).

6. Organiser une autre séance au congrès de l'ACIO/CANO de l'an prochain pour décortiquer toutes les strates de la navigation et déterminer le rôle que peut jouer l'ACIO/CANO pour orienter la navigation par les infirmières en oncologie au Canada.

7. Élaborer une politique ou un énoncé de position de l'ACIO/CANO sur la navigation.

8. Créer un groupe d'intérêt spécial sur la navigation pour faire avancer cette discussion.

\begin{tabular}{|c|c|}
\hline \multicolumn{2}{|c|}{ Représentation : 29 participants de 7 provinces } \\
\hline Question & Réponses \\
\hline $\begin{array}{l}\text { Quels sont } \\
\text { les avantages } \\
\text { à confier la } \\
\text { navigation à } \\
\text { des infirmières } \\
\text { autorisées? }\end{array}$ & $\begin{array}{l}\text { Elles peuvent offrir des services plus complets, réduire les temps d'attente et offrir du soutien psychosocial. } \\
\text { C'est particulièrement important pour les cas complexes (ex. les patients atteints de cancer de la tête et du cou), car la NPC } \\
\text { leur permet d'être vus rapidement et d'éviter qu'ils tombent entre les mailles du filet. } \\
\text { L'infirmière de référence est habituellement rattachée à un lieu en particulier, tandis que l'infirmière pivot est (souvent) } \\
\text { présente pendant toute la trajectoire du cancer, du diagnostic à la survivance, aux différents points de transition et aux passages } \\
\text { d'un milieu à un autre. Elle assume ses fonctions pendant la transition et n'est rattachée à aucun endroit particulier; elle peut } \\
\text { donc voir le patient après son départ de l'hôpital. Elle peut se mettre à l'avant-plan, se retirer, ou se mettre à l'arrière-plan à } \\
\text { tout moment de la trajectoire des soins. } \\
\text { Il y a une différence entre les grands et les petits centres, où le rôle d'intervenant pivot est plus clair, puisque c'est le seul point } \\
\text { de contact, le lien qui tient ensemble tous les morceaux. } \\
\text { L'intervenant pivot est vu comme un sauveur, pourtant on ne fait pas appel à lui autant qu'on pourrait le croire (certains } \\
\text { craignent qu'en offrant aux patients un accès libre à l'intervenant, sa charge de travail sera trop grande). Mais les patients } \\
\text { veulent surtout être rassurés et savoir que quelqu'un sera là en cas de besoin. }\end{array}$ \\
\hline
\end{tabular}


Tableau 4 : Thèmes ressortant de la première rencontre du groupe de travail en avril 2017

\begin{tabular}{|c|c|c|c|c|}
\hline Thème & $\begin{array}{l}\text { Répondre aux } \\
\text { demandes complexes } \\
\text { et diverses des } \\
\text { patients ayant des } \\
\text { besoins variables }\end{array}$ & $\begin{array}{l}\text { Assurer la continuité sur toute la } \\
\text { trajectoire des soins, de la période } \\
\text { précédant le traitement jusqu'à la } \\
\text { survivance }\end{array}$ & $\begin{array}{l}\text { Répondre aux demandes qu'on } \\
\text { ne sait où adresser à cause du } \\
\text { flou entre les limites propres } \\
\text { à chaque rôle dans l'équipe } \\
\text { soignante, et régler les tensions } \\
\text { causées par les recoupements } \\
\text { entre les diverses disciplines }\end{array}$ & $\begin{array}{l}\text { Définir clairement les limites de } \\
\text { la navigation des patients atteints } \\
\text { de cancer }\end{array}$ \\
\hline
\end{tabular}


1. Quels sont les thèmes ou éléments communs de la navigation qui sont repris dans la version préliminaire de l'énoncé de position?

2. Quels sont les éléments de la navigation qui diffèrent d'une province ou d'un programme à l'autre?

3. Manque-t-il des éléments dans l'énoncé de position qui pourraient aider le lecteur à comprendre ce qu'est la navigation des patients atteints de cancer?

4. Quelle est votre définition de la navigation des patients atteints de cancer?

Les thèmes nommés par les membres de l'équipe sont rapportés au tableau 5 .

À la lumière de ces réponses, l'énoncé de position a été révisé pour y inclure les thèmes et les expériences des participants de partout au Canada. Après la rencontre, les changements apportés à l'énoncé de position visaient notamment à préciser les liens entre la NPC et les soins centrés sur la personne; à clarifier le fait que, bien que l'objectif du programme définisse le rôle de l'infirmière pivot, les compétences et habiletés requises pour jouer ce rôle étaient similaires; à confirmer que les résultats des programmes de NPC dépendent directement de la portée du programme. Une fois l'énoncé de position lu et commenté par chaque participant, nous nous sommes réunis de nouveau pour passer l'énoncé en revue et planifier une table ronde au congrès national de 2018.

\section{8 : Table ronde au congrès de l'ACIO/CANO}

En octobre 2018, nous avons organisé une table ronde au congrès tenu à l'île-du-Prince-Édouard pour présenter à un auditoire en provenance des quatre coins du pays les progrès de l'élaboration de l'énoncé de position et le travail du groupe national. Le résumé de la séance figurait au programme : toutes les infirmières en oncologie présentes au congrès et intéressées par la NPC étaient donc invitées à s'y joindre. L'objectif de la table ronde était de souligner le travail accompli jusque-là dans la rédaction de l'énoncé de position et d'examiner l'énoncé avec les participants.

Vingt-cinq personnes provenant de partout au Canada étaient présentes et ont pris part à la discussion. La rencontre était animée par Linda Watson, qui a d'abord amené les participants à discuter de la situation de la NPC au Canada, puis des rôles actuels NPC et des progrès du groupe de travail. La discussion a récapitulé nombre de thèmes et réaffirmé à notre équipe la forte concordance entre l'énoncé de position et l'opinion de nombreux membres de l'ACIO/CANO. Nous avons aussi constaté l'évolution des rôles de navigation au pays, le nombre d'intervenants pivots ayant augmenté depuis 2016.

Les participants ont ensuite passé en revue l'énoncé de position avec les autres personnes assises à leur table. Chaque table recevait l'énoncé et Linda Watson guidait la discussion sur les points forts et les lacunes du document. Pour les participants, les éléments les plus pressants étaient de clarifier le fait que la NPC est bel et bien un rôle des infirmières spécialisées en oncologie et de décrire la NPC en termes souples, car le rôle d'infirmière pivot varie d'un poste à l'autre, même s'il existe clairement des compétences fondamentales communes. Les participants ont constaté que le document ne précisait pas la manière dont les patients pouvaient obtenir les services de l'infirmière pivot et comment le suivi prenait fin; ils ont donc invité le groupe de travail à se pencher sur cette question. Une fois ces lacunes comblées, le groupe était d'avis que l'énoncé serait prêt à être soumis au comité de pratique professionnelle de l'ACIO/CANO pour approbation et pourrait devenir, en définitive, un outil important pour guider la pratique de la NPC.

\section{DISCUSSION}

La préparation de l'énoncé de position sur la navigation de l'ACIO/CANO a nécessité plusieurs années de consultation et de collaboration avec les intervenants de partout au pays. Le congrès de l'ACIO/CANO a servi de plateforme nationale pour inviter les principaux leaders et fournisseurs de services de navigation du Canada à discuter pour mieux comprendre l'état actuel de la navigation et de ses rôles connexes. À la suite des premières discussions, la conversation a naturellement évolué vers une compréhension collective de la NPC au Canada et du rôle que peut jouer l'ACIO/CANO pour orienter et appuyer la NPC afin qu'elles soient confiées à des infirmières spécialisées en oncologie.

Grâce à ce processus pluriannuel, le rôle des infirmières pivots canadiennes a gagné en visibilité. Toutefois, des tensions persistent quant aux distinctions entre la navigation et la coordination des soins. Nous espérons que notre travail aura montré que le rôle de NPC dépasse l'aspect logistique de la coordination des soins et qu'il tient compte à la fois de la complexité des patients et du concept de soins holistiques centrés sur la personne. Certains s'inquiètent que le fait de vouloir confier le rôle de NPC à des infirmières pivots ne soit qu'un « pansement » sur un système malade et non viable et repousse la recherche de solutions aux problèmes qui plombent le système (Thorne et Truant, 2010). Au cours des vingt dernières années, le rôle de l'infirmière pivot a beaucoup évolué au Canada et dépasse maintenant les problèmes d'accès au système; il est devenu un aspect important des soins infirmiers holistiques centrés sur le patient en oncologie.

Le groupe de travail qui a organisé les ateliers de l'ACIO/ CANO en 2016 et 2017 a servi de plateforme à l'élaboration de l'énoncé de position national. Sa structure virtuelle a permis d'accélérer le travail et de poursuivre la consultation nationale. Sous la direction des meneurs du groupe de travail, les membres recevaient des tâches précises à accomplir avant chaque réunion virtuelle. Les conversations gagnaient donc en efficacité et les membres avaient en tête une orientation et un objectif clairs. La dernière rencontre et discussion dirigée par les responsables du groupe de travail (qui a eu lieu en personne) a visé à conformer la dimension nationale de l'énoncé de position et à préparer les étapes à venir, notamment la présentation au comité de pratique professionnelle de l'ACIO/ CANO pour examen et approbation.

Élément clé de l'élaboration de l'énoncé de position, le groupe de travail doit son existence à l'ACIO/CANO, qui lui a fourni une plateforme de rencontre virtuelle. La personne dirigeant l'équipe, de par ses conseils structurés, a permis au groupe de rester concentré sur ses objectifs en guidant l'analyse des commentaires formulés sur le contenu et 
Tableau 5. Réponses du groupe de travail sur la navigation à la réunion de mai 2017 : points de vue sur la version préliminaire de l'énoncé de position

\begin{tabular}{|c|c|c|c|}
\hline $\begin{array}{l}\text { Quels sont les thèmes et éléments communs de } \\
\text { la navigation qui servent de fil conducteur au } \\
\text { document? }\end{array}$ & $\begin{array}{l}\text { Quels éléments de la navigation } \\
\text { diffèrent d'une province et d'un } \\
\text { programme à l'autre? Qu'est-ce qui } \\
\text { vous semble important? }\end{array}$ & $\begin{array}{l}\text { Selon votre expérience, } \\
\text { y a-t-il des éléments } \\
\text { manquants qui aideraient } \\
\text { le lecteur à comprendre } \\
\text { la NPC? }\end{array}$ & $\begin{array}{l}\text { Quelle est votre } \\
\text { définition de la } \\
\text { navigation des patients } \\
\text { atteints de cancer? }\end{array}$ \\
\hline 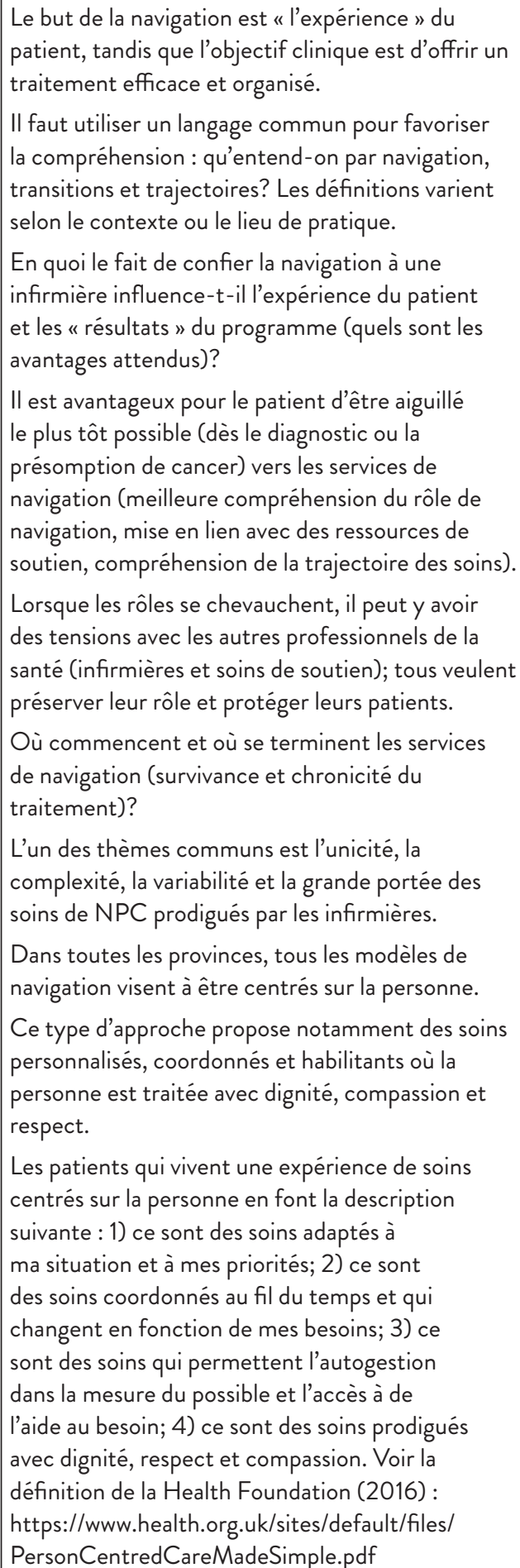 & 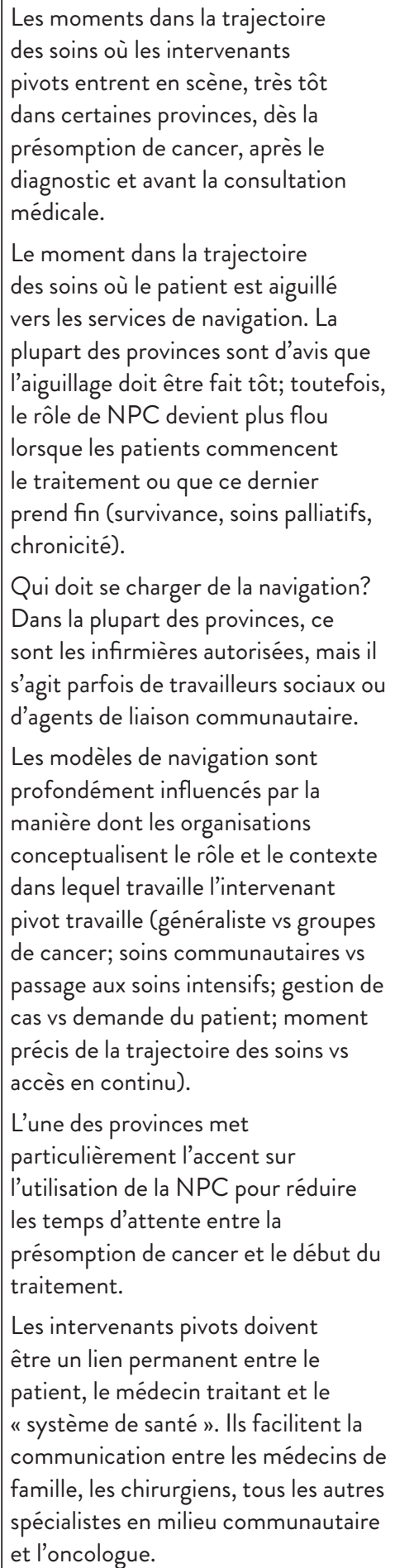 & $\begin{array}{l}\text { Dans chaque province, } \\
\text { «l'aspect " que revêt } \\
\text { la NPC est largement } \\
\text { influencé par les besoins } \\
\text { ou les lacunes que } \\
\text { l'organisation cherche à } \\
\text { combler par la NPC. Le } \\
\text { contexte de pratique de } \\
\text { la NPC influence les tâches } \\
\text { à accomplir et les objectifs à } \\
\text { atteindre. } \\
\text { Les défis se ressemblent } \\
\text { beaucoup d'un bout à } \\
\text { l'autre du pays (clarté du } \\
\text { rôle, intégration à une } \\
\text { équipe). } \\
\text { Plusieurs ressemblances } \\
\text { avec le rôle des infirmières } \\
\text { en pratique avancée et } \\
\text { la manière dont elles } \\
\text { s'intègrent à une équipe. } \\
\text { Souvent, les organisations } \\
\text { ne prennent pas le temps } \\
\text { de bien réfléchir à leur } \\
\text { approche d'intégration de } \\
\text { la NPC à l'équipe et aux } \\
\text { besoins et lacunes que le } \\
\text { rôle viendra combler, de } \\
\text { même qu'à l'organisation du } \\
\text { rôle de NPC. } \\
\text { Il faut beaucoup de temps } \\
\text { pour mettre en place un } \\
\text { nouveau rôle. Peut-être } \\
\text { qu'un cadre comme celui } \\
\text { du processus participatif, } \\
\text { basé sur des données } \\
\text { probantes et axé sur le } \\
\text { patient pour les infirmières } \\
\text { aiderait-il d'autres instances } \\
\text { intéressées par le rôle de } \\
\text { navigation à comprendre } \\
\text { comment l'intégrer dans un } \\
\text { cadre donné, de manière à } \\
\text { mieux clarifier le rôle dès le } \\
\text { départ. }\end{array}$ & $\begin{array}{l}\text { De mon côté, j'aime } \\
\text { la définition de la } \\
\text { navigation du PCCC. } \\
\text { Elle est assez générale } \\
\text { pour s'appliquer à } \\
\text { plusieurs modèles de } \\
\text { navigation, mais rend } \\
\text { aussi compte de la } \\
\text { valeur qu'apporte la } \\
\text { navigation. } \\
\text { Il s'agit d'une } \\
\text { personne désignée } \\
\text { dans l'équipe de soins } \\
\text { ayant la capacité de } \\
\text { se concentrer sur les } \\
\text { besoins en navigation } \\
\text { des patients et des } \\
\text { familles. Elle peut avoir à } \\
\text { aider les autres systèmes } \\
\text { et professionnels à } \\
\text { répondre aux besoins } \\
\text { des patients. } \\
\text { Les programmes } \\
\text { de NPC visent surtout } \\
\text { à répondre aux besoins } \\
\text { des patients en } \\
\text { optimisant les processus } \\
\text { du système plutôt qu'en } \\
\text { répondant d'abord aux } \\
\text { besoins du système, les } \\
\text { faisant passer devant } \\
\text { ceux des patients. }\end{array}$ \\
\hline
\end{tabular}


l'articulation de l'énoncé de position. Cette approche a facilité la rédaction de l'énoncé, mais elle a tout de même exigé plusieurs années de travail. Il n'est pas simple de réunir virtuellement de multiples intervenants de partout au pays lorsqu'il faut jongler avec les horaires de chacun, le choix du moment de la rencontre, ainsi que la gestion et l'utilisation des plateformes virtuelles.

Pour la suite des choses, un groupe d'intérêt spécial a été formé pour animer au congrès de 2019 un atelier axé sur la création et la mise à jour de modules de formation ayant pour but de préciser d'améliorer les habiletés et compétences requises pour jouer le rôle de NPC, le tout orienté par l'énoncé de position rédigé par le groupe de travail.

\section{RÉFÉRENCES}

Braun, V., \& Clarke, V. (2008). Using thematic analysis in psychology. Qualitatve Research in Psychology, 3(2), 24.

Campbell, C., Craig, J., Eggert, J., \& Bailey-Dorton, C. (2010). Implementing and measuring the impact of patient navigation at a comprehensive community cancer center. Paper presented at the Oncology Nursing Forum.

Canadian Association of Nurses in Oncology. (2001). The standards of care.http://www.cano-acio.ca/en/ practice/standards_of_care.html

Canadian Partnership Against Cancer. (2010). Guide to implementating navigation. Author.

Cantril, C., \& Haylock, P. J. (2013). Patient navigation in the oncology care setting. Seminars in Oncology Nursing, 29(2), 14. https://doi. org/10.1016/j.soncn.2013.02.003

Case, M. A. B. (2011). Oncology nurse navigator. Clinical Journal of Oncology Nursing, 15(1).

Cook, S., Fillion, L., Fitch, M. I., Veillette, A.-M., Matheson, T., Aubin, M., ... Rainville, F. (2013). Core areas of practice and associated competencies for nurses working as professional cancer navigators. Canadian Oncology Nursing Journal/Revue canadienne de soins infirmiers en oncologie, 23(1), 44-52.

Doll, R., Barroetavena, M. C., Ellwood, A.-L., Fillion, L., Habra, M., \& Linden, W. (2007). The cancer care navigator. Toward a conceptual framework for a new role in oncology. Oncology Exchange, 6(4), 28-33.

Ferrell, B., McCabe, M. S., \& Levit, L. (2013). The Institute of Medicine report on high-quality cancer care: implications for oncology nursing. Paper presented at the Oncology Nursing Forum.

Fillion, L., Cook, S., Veillette, A.-M., Aubin, M., De Serres, M., Rainville, F., ... Doll, R. (2012). Professional navigation framework: Elaboration and validation in a Canadian context. Oncology Nursing Forum, 39(1). https://doi.org/10.1188/12.ONF.E58-E69

Fillion, L., de Serres, M., Cook, S., Goupil, R. L., Bairati, I., \& Doll, R. (2009). Professional patient navigation in head and neck cancer. Seminars in Oncology Nursing, 25(3), 212-221.

Fillion, L., de Serres, M., Lapointe-Goupil, R., Bairati, I., Gagnon, P., Deschamps, M., ... Demers, G. (2006). Implementing the role of a patient-navigator nurse at a university hospital centre. Canadian Oncology Nursing Journal, 16, 11-17.

Freund, K. (2011). Patient navigation: The promise to reduce health disparities. Journal of General Internal Medicine, 26(2), 110-112. http://dx.doi.org/10.1007/s11606-010-1593-5

Hébert, J., \& Fillion, L. (2011). Gaining a better understanding of the

\section{CONCLUSIONS}

Depuis le début des travaux du groupe en 2016, le paysage de la navigation a évolué partout au Canada. Le rôle de NPC joué par les infirmières en oncologie est maintenant bien implanté dans des provinces ou territoires où il n'était pas présent auparavant, et il continue de pourvoir à des besoins essentiels des patients à tous les points de la trajectoire du cancer. Il y a encore du travail à faire pour sonder la perception des patients ayant bénéficié de soins de navigation. Grâce aux travaux menés d'un bout à l'autre du pays pendant toutes ces années, nous avons la possibilité d'appuyer les différents modèles de NPC et de comprendre leur influence sur les résultats et les expériences rapportés par les patients.

support function of oncology nurse navigators from their own perspective and that of people living with cancer: Part 2. Canadian Oncology Nursing Journal/Revue canadienne de soins infirmiers en oncologie, 21(2), 7.

McMullan, M. (2006). Patients using the Internet to obtain health information: How this affects the patient-health professional relationship. Patient Education and Couselling, 63(12), 24-28.

Ministère de la Santé et des Services sociaux (MSSS). (2005). Programme québécois de lutte contre le cancer. Pour optimiser la contribution des infirmières à la lutte contre le cancer. Comité de l'évolution de la pratique infirmière en oncologie (CEPIO). www.msss. gouv.qc.ca/.../optimiser_contribution_infirmieres_mars2005.pdf

Ministère de la santé et des services sociaux (MSSS). (2008). Programme québécois de lutte contre le cancer. Rôle de l'infirmière pivot en oncologie. Comité consultatif des infirmières en oncologie. www. msss.gouv.qc.ca/inc/documents/ministere/lutte-contre-le-cancer/ role-infirmiere-pivot_juil2008.pdf

Pedersen, A. E., Hack, T. F., McClement, S. E., \& Taylor-Brown, J. (2014). An exploration of the patient navigator role: Perspectives of younger women with breast cancer. Oncology Nursing Forum, 41(1), 11.

Pedersen, A. F., \& Hack, T. F. (2010). Pilots of oncology health care: A concept analysis of the patient navigator role. Oncology Nursing Forum, 37(1), 55-60. https://doi.org/10.1188/10.onf.55-60

Pratt-Chapman, M., Simon, M. A., Patterson, A. K., Risendal, B. C., \& Patierno, S. (2011). Survivorship navigation outcome measures. Cancer, 117(S15), 3573-3582. http://dx.doi.org/10.1002/cncr.26261

Seek, A., \& Hogle, W. (2007). Modeling a better way: Navigating the healthcare system for patients with lung cancer. Clinical Journal of Oncology Nursing, 11(1). https://doi.org/10.1188/07.CJON.81-85

Thorne, S., \& Truant, T. (2010). Will designated patient navigators fix the problem? Oncology nursing in transition. Canadian Oncology Nursing Journal/Revue canadienne de soins infirmiers en oncologie, 20(3), 116-121.

Watson, L. C., Vimy, K., Anderson, J., Champ, S., \& DeIure, A. (2016). Developing a provincial cancer patient navigation program utilizing a quality improvement approach. Part three: Evaluation and outcomes. Canadian Oncology Nursing Journal, 26(4), 276. 